\title{
Papilas Fungiformes Pigmentadas de la Lengua. Características Clínicas, Histológicas y Dermatoscópicas de una Serie de Casos Ecuatorianos
}

\author{
Pigmented Fungiform Papillae of the Tongue. Clinical, Histological \\ and Dermatoscopic Characteristics A Serie of Ecuadorian Cases
}

Viviana Garzón Rivas \& Eduardo Garzón Aldás

\begin{abstract}
GARZÓN, R. V. \& GARZÓN, A. E. Papilas fungiformes pigmentadas de la lengua. Características clínicas, histológicas y dermatoscópicas de una eerie de casos ecuatorianos. Int. J. Odontostomat., 13(4):442-445, 2019.

RESUMEN: Las papilas fungiformes pigmentadas de la lengua, cuyas siglas son PFPT, del inglés Pigmented fungiform papillae of the tongue, es una condición asintomática, no progresiva que se presenta en personas de piel oscura, en las cuales las papilas fungiformes cambian de su color rosado natural, a una gama de café a negro. El objetivo de nuestro estudio es reportar y describir las caractetísticas clínicas, dermatoscópicas e histológicas de la PFPT por primera vez en una serie de pacientes ecuatorianos. Estudio prospectivo simple en el Centro de Especialidades Dermatológicas Garzón, período de dos años. El criterio de inclusión fue cambio de coloración a nivel lingual, se recolectaron datos demográficos, clínicos; fotografías, dermatoscopía, y biopsia, para tinción con hematoxilina-eosina y Fontana-Mason. Examinamos 8.640 pacientres, 15 (12 mujeres, 3 varones) fueron diagnosticados de PFPT. La edad promedio fue 31 años, todos fueron mestizos, con fototipo de piel predominante III y IV. El tiempo de evolución promedio en años fue 5,8. Ninguno tuvo antescedentes familiares o personales relacionados a la patología. La evaluación clínica demostró que el patrón de distribución de acuerdo a la clasificación de Holzwanger en la gran mayoría fue tipo Il (13/15). En todos los casos la dermatoscopía y la histología fueron específicas demostrando hallazgos típicos y comprobatorios de PFPT.
\end{abstract}

PALABRAS CLAVE: papilas fungiformes, lengua, hiperpigmentación, dermatosocopía.

\section{INTRODUCCIÓN}

En la superficie lingual encontramos tres tipos de papilas: fungiformes, filiformes y circunvaladas. Las primeras están ubicadas en la punta y en los aspectos laterodorsales de la lengua, interviniendo en las funciones gustativas (Oh et al., 2000).

Las papilas fungiformes pigmentadas de la lengua, cuyas siglas son PFPT, del inglés Pigmented fungiform papillae of the tongue, fueron descritas por primera vez por Leonard (1905), como una condición asintomática, no progresiva que se presenta entre la segunda y tercera década de vida, en la lengua de personas de piel oscura, sin embargo existen reportes de casos en la infancia, así como en poblaciones Japonesas, Chinas e Indúes, en las cuales las papilas fungiformes cambian de su color rosado natural, a una gama de café a negro. El objetivo de nuestro estudio es reportar y describir las caractetísticas clínicas, dermatoscópicas e histológicas de la PFPT por primera vez en una serie de pacientes ecuatorianos.

\section{MATERIAL Y MÉTODO}

Realizamos un estudio prospectivo simple en pacientes que acudieron a la consulta del Centro Dermatológico Garzón, Quito-Ecuador, en el período comprendido entre enero 2017 a enero 2019. El criterio de inclusión fue cambio de coloración a nivel lingual en pacientes de todas las edades y sexo. Realizamos recolección de datos demográficos y clínicos como: edad, sexo, raza, fototipo, historia familiar similar, tiempo de evolución, morfología, distribución, extensión, signos/ 
síntomas acompañantes; tomamos fotografías clínicas, realizamos dermatoscopía a todos los pacientes y la documentamos fotográficamente, y por último tomamos biopsia por punch $3 \mathrm{~mm}$, previo consentimiento informado de los pacientes, para tinción con hematoxilinaeosina, y Fontana-Masson.

\section{RESULTADOS}

Pacientes: Sexo, Edad, Raza, fototipo, tiempo evolución, historia familiar, historia patológica personal. Nosotros examinamos un total de 8.640 pacientres, de los cuales 15 ( 12 mujeres, 3 varones) consultaron por cambios de coloración en mucosa lingual que correspondió al diagnóstico de PFPT. La edad promedio fue 31 años (rango entre 8 a 52). Todos fueron mestizos, con fototipo de piel predominante III y IV. El tiempo de evolución promedio en años fue 5,8 (rango entre 1 a 20), y en ninguno se encontró historia familiar similar. Los antecedentes personales no fueron relevantes, ninguno había consumido fármacos o sustancias relacionadas con pigmentación como tetraciclinas o sus derivados, antipalúdicos entre otros; y solo en 3 pudimos evidenciar restauraciones dentales con amalgama, en el resto hubo restauraciones con resina (Tabla I).

Morfología, distribución, extensión, signos/síntomas acompañantes. El exámen clínico, en este estudio, se lo hizo, considerando la clasificación de Holzwanger et al. (1974), el cual describe tres patrones de PFPT: Tipo I: se caracteriza por un parche bien circunscrito en el que se ven afectadas todas las papilas fungiformes en el área, ubicada ya sea en el aspecto anterior-lateral o en la punta de la lengua; Tipo
II: hiperpigmentación que involucra de 3 a 7 papilas fungiformes dispersas o ligeramente agrupadas en el dorso lingual con concurrencia de papilas fungiformes y filiformes normales ; Tipo III: hiperpigmentación completa de cada una de las papilas fungiformes en el dorso de la lengua.

En nuestra serie de casos se observaron pápulas puntiformes de color marrón oscuro a negro en todos los casos; la evaluación de su distribución mostró que 2/15 pacientes presentaron un patrón tipo I (Fig. 1); 13/15 pacientes presentaron patrón tipo II (Fig. 2); y ninguno patrón tipo III.

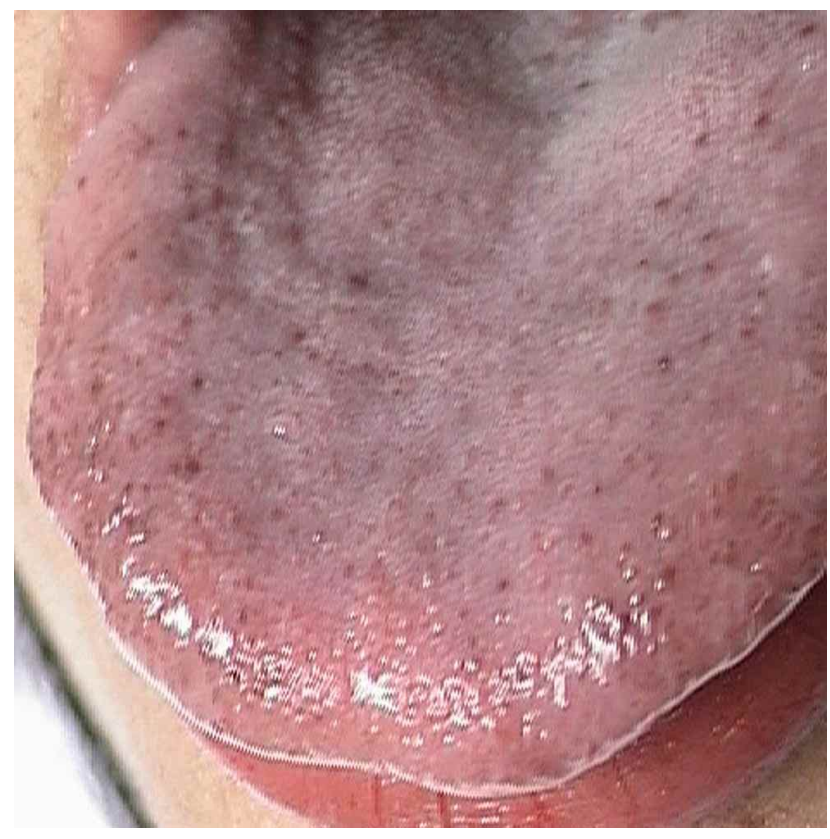

Fig. 2. Patrón de pigmentación tipo II, paciente femenina. Foto panorámica, obsérvese varias papilas fungiformes hiperpigmentadas dispersas en el dorso de la lengua.

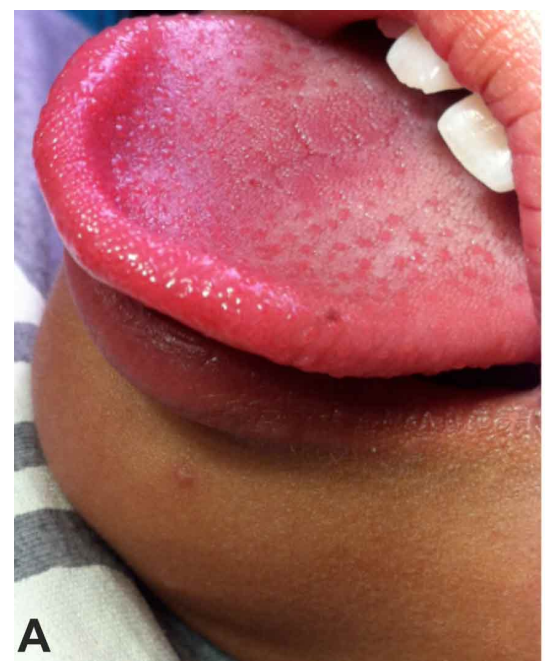

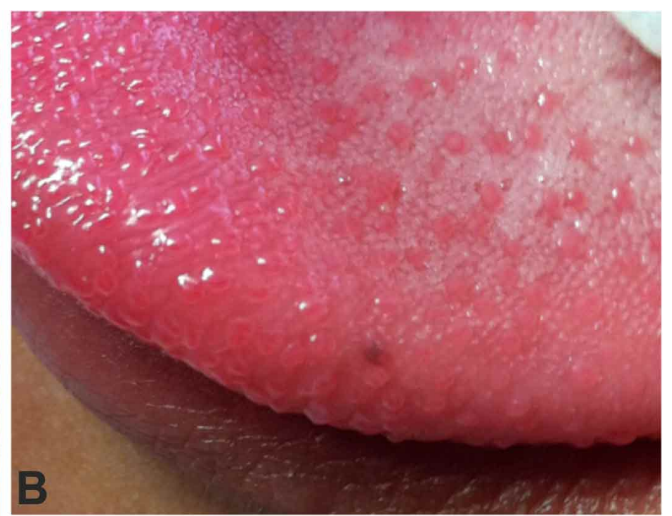

Fig. 1. A Patrón de pigmentación tipo I en paciente pediátrico, foto panorámica. B. Acercamiento que muestra lesión única hiperpigmentada en margen antero-lateral de la lengua. 
Un examen físico completo no encontró asociación con hiperpigmentación de otros sitios cutáneos, ungueales o mucosos (Tabla I).

Dermatoscopía. La dermatoscopía (DS) fue realizada con un dermatoscopio de luz polarizada Canfield VEOS HD2 y documentadas con el mismo sistema fotográfico asociado a su app para instrumentos Apple . DS mostró en la totalidad de pacientes papilas con patrón hiperpigmentado en "empedrado o adoquinado" (Fig. 3 A). En cada papila se detectaron proyecciones con márgenes pigmentados, intercaladas por vasos sanguíneos dicotomizados originados en su base; mostrando un patrón típico de "pétalo de rosa" (Fig. 3B)

Histología. Se tomó muestra de mucosa lingual para biopsia mediante punch de $3 \mathrm{~mm}$, y se realizó tinción para hematoxilina-eosina, Fontana-Masson. El examen histológico de los quince pacientes reveló melanófagos subepiteliales con infiltrado linfocítico leve en el corion superficial (Fig. 4). El pigmento dentro de los melanófagos se mostró positivo para melanina con tinción de Fontana-Masson en todos los casos.

Tabla I. Datos demográficos y clínicos de los pacientes con PFPT.

\begin{tabular}{|c|c|c|c|c|c|c|c|}
\hline Paciente & Edad & Sexo & Fototipo & Raza & T.Evoluc. & $\begin{array}{l}\text { Patrón } \\
\text { clínico }\end{array}$ & $\begin{array}{l}\text { Pigmentación en } \\
\text { mucosas o uñas }\end{array}$ \\
\hline 1 & 8 & $\mathrm{M}$ & III & Mestiza & 1 & 1 & No \\
\hline 2 & 36 & $\mathrm{~F}$ & III & Mestiza & 2 & II & No \\
\hline 3 & 40 & $\mathrm{~F}$ & III & Mestiza & 5 & II & No \\
\hline 4 & 18 & $\mathrm{~F}$ & III & Mestiza & 1 & II & No \\
\hline 5 & 22 & $\mathrm{~F}$ & IV & Mestiza & 3 & II & No \\
\hline 6 & 39 & $\mathrm{~F}$ & III & Mestiza & 6 & II & No \\
\hline 7 & 51 & $\mathrm{~F}$ & IV & Mestiza & 17 & II & No \\
\hline 8 & 48 & $F$ & III & Mestiza & 10 & II & No \\
\hline 9 & 24 & M & III & Mestiza & 8 & I & No \\
\hline 10 & 27 & $\mathrm{~F}$ & III & Mestiza & 6 & II & No \\
\hline 11 & 18 & $\mathrm{~F}$ & IV & Mestiza & 1 & II & No \\
\hline 12 & 31 & $\mathrm{~F}$ & III & Mestiza & 1 & II & No \\
\hline 13 & 29 & $\mathrm{~F}$ & III & Mestiza & 4 & II & No \\
\hline 14 & 26 & $\mathrm{~F}$ & IV & Mestiza & 3 & II & No \\
\hline 15 & 52 & $M$ & III & Mestiza & 20 & II & No \\
\hline
\end{tabular}

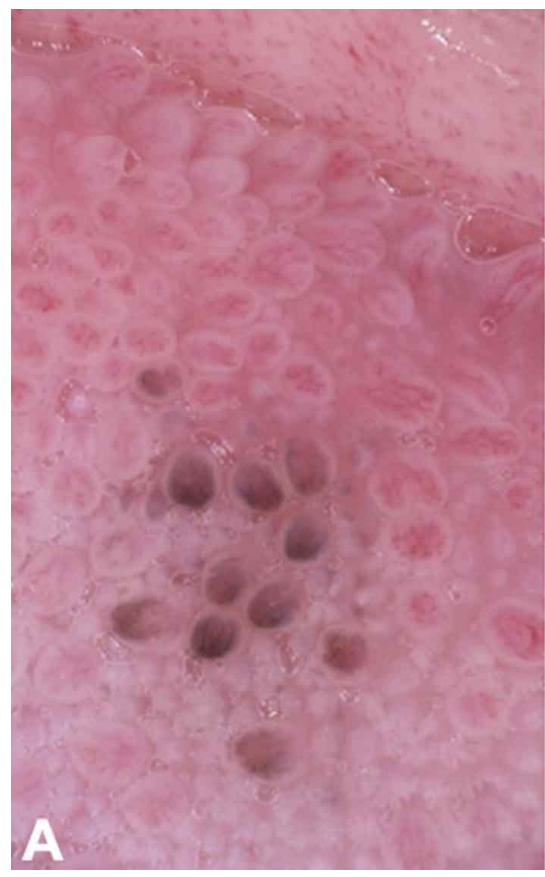

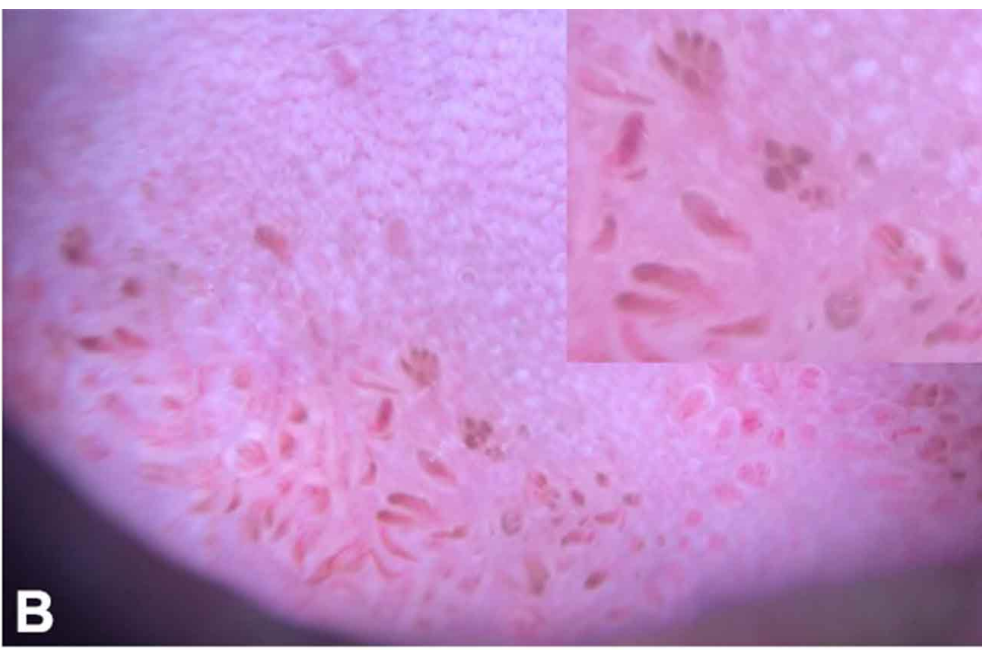

Fig. 3. Fotos dermatoscópicas en las que se demuestra: A. patrón hiperpigmentado en "empedrado o adoquinado" paciente \# 1. B. En cada papila se detectaron proyecciones con márgenes pigmentados, vasos sanguíneos dicotomizados mostrando un patrón en "pétalo de rosa". En la esquina superior derecha gran acercamiento, paciente \# 10. 


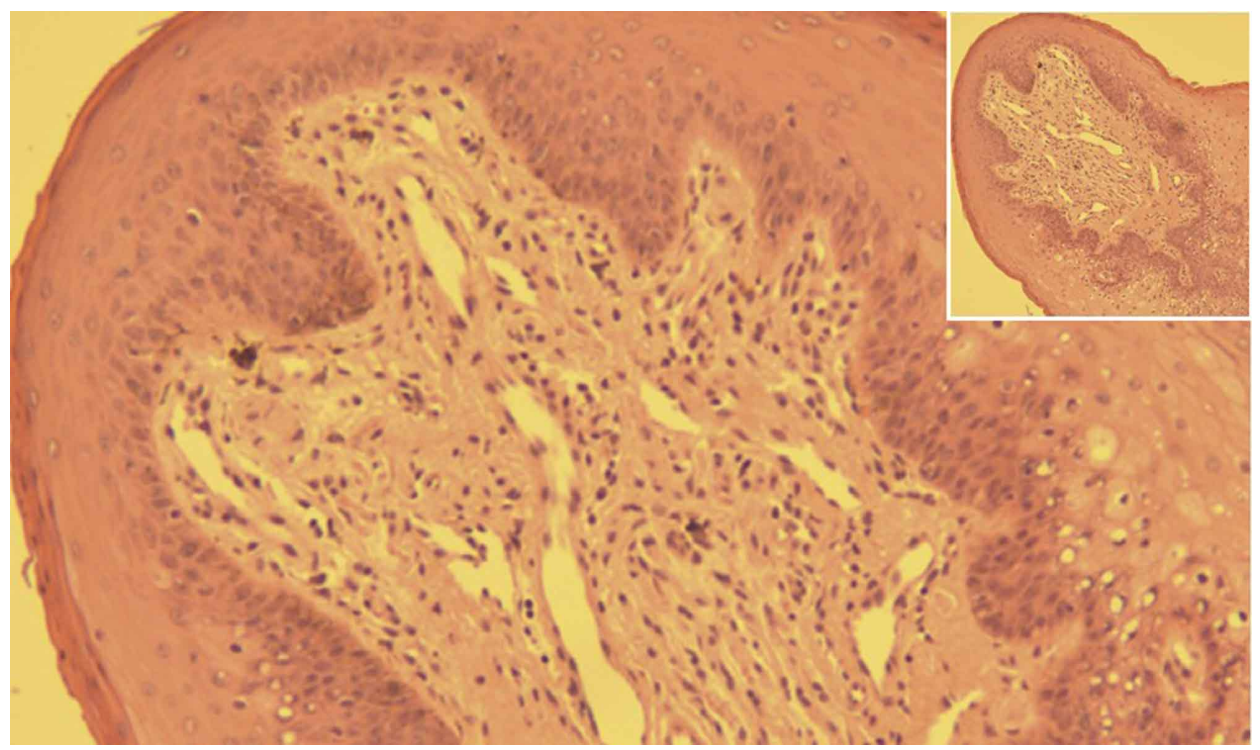

Fig. 4. Histopatología de PFPT. Tinción H-E. 10 x. Se observan melanófagos subepiteliales con infiltrado linfocítico leve en el corion superficial, espacios vasculares dilatados. En la esquina superior derecha foto de menor aumento 4x.

\section{DISCUSIÓN}

La superficie de la lengua consta de tres tipos de papilas, que se denominan filiformes, fungiformes y circunvaladas (Lin \& Chou, 2013). Las papilas filiformes se distribuyen uniformemente sobre los dos tercios anteriores del dorso de la lengua. Las papilas circunvaladas son estructuras en forma de domo situadas en el dorso de la lengua inmediatamente delante del foramen ciego y el surco terminal. Las papilas fungiformes son proyecciones rosadas discretas, concentradas en los márgenes de la lengua y dispersas entre todas las papilas filiformes, aunque están ubicadas principalmente en la punta y los márgenes laterales de la lengua, (Adibi et al., 2011). PFPT describe una variante negra o marrón de papilas fungiformes que se observa comúnmente en razas muy pigmentadas (Tan et al., 2014).

Leonard fue el primero en reportar PFPT en 1905. En 1967, Koplon y Hurley realizaron un estudio retrospectivo y observaron PFPT en 2 mujeres negras, e informaron una incidencia de PFPT del $0,4 \%$ en personas de raza negra y $0 \%$ en caucásicos (Romiti \& Molina De Medeiros, 2010). En 1973, Holzwanger et al. examinaron una muestra aleatoria de 300 individuos, encontrando que la PFPT tenía una prevalencia de casi el $30 \%$ en mujeres negras y el $25 \%$ en hombres de la misma raza. A lo largo de los años se ha reportado en la literatura casos de PFPT en asiáticos, hispanos, y en caucásicos (Robles-Méndez et al., 2017). En nuestra serie de casos (15/15) todos fueron mestizos. Así se ha demostrado la mayor incidencia de PFPT en personas de raza negra. Koplon y otros investigadores estimaron que ocurre PFPT en menos de 1 de cada 20000 pacientes ambulatorios dermatológicos en la India, si consideramos nuestros datos, la incidencia entre pacientes dermatológicos sería mayor ( 15 en 8.640 ) seguramente por el fototipo de piel que caracteriza a nuestra población, es decir tipo III y IV, lo que concordaría, que la patología es más frecuente en los fototipos más pigmentados.

La PFPT generalmente se desarrolla durante la segunda y tercera décadas de la vida, mientras que el inicio pediátrico se ha reportado raramente (Mangold, 2016). En nuestra serie de casos solo 1 de 15 fue pediátrico, el resto supero la tercera década de vida, coinciendo con lo reportado por otros autores.

En la práctica diaria, muchos dermatólogos rara vez exploran la cavidad oral en pacientes asintomáticos; esta es la razón por la cual la prevalencia de PFPT puede ser subestimada (Urbina \& Sudy, 2013). Hasta la fecha, se han reportado asociaciones dermatológicas casuales según Karine Francine Docx et al. (2016) con: liquen plano; ictiosis linear circunfleja; y como parte integrante del síndrome de Laugier- 
Hunziker, caracterizado por máculas pigmentadas en los labios, mucosa oral y paladar; a veces asociadas con melanoniquia; nevus de Hori; y melasma (Cinotti et al., 2017). Asociaciones sistémicas con: anquilostomiasis, deficiencia de hierro, hemocromatosis, anemia. Sin embargo, en la mayoría de los casos, los pacientes con PFPT no presentan ninguna enfermedad asociada; como en nuestros pacientes, en los cuales no se encontró ninguna otra signología dermatológica o sistémica.

Se han definido tres patrones de PFPT. El tipo I se caracteriza por un parche bien circunscrito en el que se ven afectadas todas las papilas fungiformes en el área. En el tipo II, la hiperpigmentación lingual consiste esencialmente en tres a siete papilas fungiformes que se dispersan aleatoriamente en la superficie lingual dorsal. El tipo III es menos común, con solo dos casos notificados (incluido el nuestro) (Halder \& Nootheti, 2003). En el tipo III, la hiperpigmentación afecta a casi todas las papilas fungiformes en el dorso de la lengua (Ghigliotti et al., 2017). En nuestra serie de casos predominó el patrón tipo II, seguido por el tipo I, y en ningún caso vimos el patrón tipo III; este hallazgo es consistente con los datos de la literatura, cabe mencionar además que el patrón tipo I es más frecuente en la edad pediátrica, y que en algunos casos se ha reportado superposición del patrón tipo I/tipo II (Marcoval et al., 2011).

Histopatológicamente, la PFPT muestra melanófagos prominentes en la lámina propia sin anormal sin hallazgos de daño epidérmico o infiltrado inflamatorio dérmico prominente, existen muy pocos casos reportados con focos de daño vacuolar de la basal, en asociación con liquen ruber plano (Al-Fagaan \& Joseph, 2014).

Hsiao et al. (2011) describieron un patrón dermatoscópico en empedrado en PFEPT; posteriormente, Mukamal et al. (2012) notaron que la pigmentación de cada elemento de PFPT se encontraba principalmente en las puntas de las papilas, desvaneciéndose ligeramente hacia el centro de la misma esto sugería un "patrón de pétalos de rosa". En nuestra opinión, estos términos definen: el empedrado o adoquinado es una descripción de la distribución de las papilas fungiformes pigmentadas en la lengua, mientras que el patrón de pétalos de rosa es una descripción detallada de la distribución del pigmento en la papila individual, además, en cada papila se pueden ver vasos sanguíneos dicotomizados originados en su base. Cabe destacar que los patrones clínicos y dermatoscópicos no muestran diferencias. comparando adultos y niños como se pudo ver en nuestros pacientes.

A pesar de que la etiología del PFTP aún no está clara, la activación de melanófagos subepiteliales están implicados en la hiperpigmentación de las papilas sin ninguna evidencia histológica de inflamación (Millington \& Shah, 2007). Sin embargo, en ciertos reportes se han encontrado linfocitos generando daño vacuolar de la basal con la subsecuente incontinencia del pigmento, lo que daría orígen a los melanófagos , los cuales reactivarían procesos de melanogénesis (Scarff \& Marks, 2003). Nosotros pensamos que este es un proceso evolutivo cronológico, y que la falta de estos hallazgos (daño vacuolar) en todos los casos, se debería o estaría sujeto al tiempo de evolución en el que se toma la biopsia en cada caso en particular.

Aunque la mayoría de los informes son casos esporádicos, casos intrafamiliares se han reportado en la madre como en el hijo sugiriendo un componente hereditario tipo autosómico dominante según lo informado por Werchniak et al. (2004).

Debido a que PFPT no tiene síntomas, no hay necesidad de ningún tratamiento, así como por lo que suele permanecer estacionaria, sin mostrar progresión (Collgros et al., 2015).

En conclusión presentamos la primera serie de casos ecuatorianos de papilas fungiformes pigmentadas de la lengua, la misma que es consistente clínica, dermatoscópica, e histológicamente, con lo reportado previamente en la literatura. Estamos convencidos que esta patología está infradiagnosticada, al no tener una conducta permanente de colaboración multidisciplinaria; el apoyo de dermatología / odontología / estomatología, en doble vía para la adecuada resolución de casos de patología oral es indispensable.

GARZÓN, R. V. \& GARZÓN, A. E. Pigmented fungiform papillae of the tongue. Clinical, histological and dermatoscopic characteristics a serie of ecuadorian cases. Int. J. Odontostomat., 13(4):445-451, 2019.

ABSTRACT: The pigmented fungiform papillae of the tongue, whose acronyms are PFPT, of the English Pigmented fungiform papillae of the tongue, is an asymptomatic, nonprogressive condition that occurs in dark-skinned people, in which the fungiform papillae change their color natural pink, to a range of brown to black. The aim of our study is to report 
and describe the clinical, dermatoscopic and histological characteristics of the PFPT for the first time in a series of Ecuadorian patients. A simple prospective study at the Garzón Dermatological Specialty Center, a two-year period. The inclusion criteria was lingual change of color, demographic, clinical data were collected; photographs, dermatoscopy, and biopsy, for staining with hematoxylin-eosin and FontanaMason. We examined 8,640 patients, 15 (12 women, 3 men) were diagnosed with PFTP. The range of age was 31 years, all were mestizos, with skin phototype predominant III and IV. The range of evolution time in years was 5.8. None had family or personal precedents related to the pathology. The clinical evaluation showed that the pattern of distribution according to the Holzwanger classification in the great majority was type II (13/15). In all cases, the dermatoscopy and histology were specific, demonstrating typical and evidential findings of PFPT.

KEY WORDS: fungiform papillae, tongue, hyperpigmentation, dermatosocopy.

\section{REFERENCIAS BIBLIOGRAFICAS}

Adibi, S.; Suarez, P. \& Bouquot, J. E. Papillary tip melanosis (pigmented fungiform lingual papillae). Tex. Dent. J., 128(6):5723, 576-8, 2011.

Al-Fagaan, F. \& Joseph, B. A case of pigmented fungiform papillae of the tongue in a Middle Eastern woman. Med. Princ. Pract., 23(2):167-9, 2014

Cinotti, E.; Labeille, B.; Cambazard, F. \& Perrot, J. L. Dermoscopy and reflectance confocal microscopy examination of pigmented fungiform papillae of the tongue. Ann. Dermatol. Venereol., 144(4):323-5, 2017.

Collgros, H.; Iglesias-Sancho, M. \& Galvany, L. Dermoscopy of pigmented fungiform papillae of the tongue in an Indian girl. Australas. J. Dermatol., 56(3):e81-2, 2015.

Ghigliotti, G.; Chinazzo, C.; Parodi, A. \& Rongioletti, F. Pigmented fungiform papillae of the tongue: the first case in an Italian woman. Clin. Exp. Dermatol., 42(2):206-8, 2017.

Halder, R. M. \& Nootheti, P. K. Ethnic skin disorders overview. J. Am. Acad. Dermatol., 48(6 Suppl.):S143-8, 2003.

Holzwanger, J. M.; Rudolph, R. I. \& Heaton, C. L. Pigmented fungiform papillae of the tongue: a common variant of oral pigmentation. Int. J. Dermatol., 13(6):403-8, 1974.

Hsiao, Y. H.; Ko, J. H.; Lu, C. F. \& Chen, M. J. Dermoscopic findings in pigmented fungiform papillae of the tongue. Eur. J. Dermatol., 21(5):819-20, 2011.

Karine Francine Docx, M.; Vandenberghe, P. \& Govaert, P. Pigmented fungiform papillae of the tongue (PFPT). Acta Clin. Belg., 71(2):117-8, 2016.

Leonard, T. M. R. Ankylostomiasis or uncinariasis. JAMA, 45(9):58894, 1905.

Lin, Y. T. \& Chou, C. L. Pigmented macule on the tongue of a 12year-old girl. J. Am. Acad. Dermatol., 69(5):e229-30, 2013.

Mangold, A. R.; Torgerson, R. R. \& Rogers, R. S. 3rd. Diseases of the tongue. Clin. Dermatol., 34(4):458-69, 2016.

Marcoval, J.; Notario, J.; Martín-Sala, S. \& Figueras, I. Pigmentation of the fungiform papillae of the tongue: a report of 2 cases. Actas Dermosifiliogr., 102(9):739-40, 2011.
Millington, G. W. \& Shah, S. N. A case of pigmented fungiform lingual papillae in an Indian woman. J. Eur. Acad. Dermatol. Venereol., 21(5):705, 2007.

Mukamal, L. V.; Ormiga, P. \& Ramos-E-Silva, M. Dermoscopy of the pigmented fungiform papillae of the tongue. J. Dermatol., 39(4):397-9, 2012.

Oh, C. K.; Kim, M. B.; Jang, H. S. \& Kwon, K. S. A case of pigmented fungiform papillae of the tongue in an Asian male. J. Dermatol., 27(5):350-1, 2000.

Robles-Méndez, J. C.; Ayala-Cortés, A. S.; Villarreal-Martínez, A. \& Ocampo-Candiani, J. Dermoscopy of pigmented fungiform papillae of the tongue. J. Am. Acad. Dermatol., 76(2S1):S40S42, 2017.

Romiti, R. \& Molina De Medeiros, L. Pigmented fungiform papillae of the tongue. Pediatr. Dermatol., 27(4):398-9, 2010.

Scarff, C. E. \& Marks, R. Pigmented fungiform papillae on the tongue in an Asian man. Australas. J. Dermatol., 44(2):149-51, 2003.

Tan, C.; Liu, Y.; Min, Z. S. \& Zhu, W. Y. A clinical analysis of 58 chinese cases of pigmented fungiform papillae of the tongue. $J$. Eur. Acad. Dermatol. Venereol., 28(2):242-5, 2014.

Urbina, F. \& Sudy, E. Pigmented fungiform papillae of the tongue in Laugier disease (or Laugier-Hunziker syndrome). Actas Dermosifiliogr., 104(2):173-4, 2013.

Werchniak, A. E.; Storm, C. A. \& Dinulos, J. G. Hyperpigmented patches on the tongue of a young girl. Pigmented fungiform papillae of the tongue. Arch. Dermatol., 140(10):1275-80, 2004.

Direccion para correspondencia:

Dr. Eduardo Garzón Aldás

Dermatólogo

Universidad Central Del Ecuador

ECUADOR

Email:eduderma@hotmail.com

Recibido : 22-04-2019

Aceptado: 17-07-2019 